\title{
Effects of identical context on visual pattern recognition by pigeons
}

\author{
FRANCISCO J. DONIS \\ Central Connecticut State University, New Britain, Connecticut \\ and \\ SHEILA CHASE and ERIC G. HEINEMANN \\ Hunter College, City University of New York, New York
}

\begin{abstract}
The effects of identical context on pattern recognition by pigeons for outline drawings of faces were investigated by training pigeons to identify (Experiment 1) and categorize (Experiment 2) these stimuli according to the orientation of the mouth - an upright U shape representing a smiling mouth or an inverted U shape representing a sad mouth. These target stimuli were presented alone (Pair 1), with three dots in a triangular orientation to represent a nose and eyes (Pair 2), and with the face pattern surrounded by an oval (Pair 3). In Experiment 1, the pigeons trained with Pair 1 were most accurate, those trained with Pair 2 were less so, and those trained with Pair 3 failed to acquire the discrimination within eighty 100-trial sessions. The same ordering was found in Experiment 2 for pigeons trained on the three pairs simultaneously. The authors suggest that a contrasting finding in humans, the face superiority effect, might be due to a gain in discriminability resulting from recognition of the pattern as a face. An exemplar model of information processing that excludes linguistic coding accounts for the present results.
\end{abstract}

Certain cognitive abilities, once thought to be the exclusive domain of humans, are now recognized as part of the behavioral repertoire of other animals. Of particular interest here is how pigeons categorize complex visual patterns, which may be photographic exemplars of classes humans identify by name, such as trees, fish, or water. Although Herrnstein and Loveland's seminal paper, "Complex visual concept in the pigeon," appeared in 1964, there still is no agreement as to how such stimuli are processed. Do pigeons form a prototype representative of the class of patterns, extract common features, or just remember individual patterns? (For a review, see Huber, 2001). To resolve these issues, one must be able to specify the stimulus as perceived by the pigeon. For the purposes of this research, the complexity of the visual stimuli has been reduced to simple outline forms.

Although it is convenient to assume that the pigeon sees the world as we do, this is an unwarranted assumption. Physiological and anatomical data provide valuable guidelines, but our understanding of the pigeon's view of the world requires the analysis of its perception as revealed through its behavior. The comparative analysis of pigeon and human form perception in visual search (e.g., D. S. Blough \& P. M. Blough, 1997; Cook, Cavoto, \& Cavoto,

We thank Keith Johnson, Elisa Warga, and Peter Krzykowski for their assistance in running the subjects and two anonymous reviewers for their helpful comments. Correspondence concerning this article should be addressed to F. J. Donis, Department of Psychology, Central Connecticut State University, New Britain, CT 06050-4010 (e-mail: donis@ccsu.edu); or to S. Chase or E. G. Heinemann, Department of Psychology, Hunter College, 695 Park Avenue, New York, NY 10021 (e-mail: schase@hunter. cuny.edu or eheinema@hunter.cuny.edu).
1996) represents an important step in this direction, as does other work showing similarity and differences in the behavior of these species (e.g., Hollard \& Delius, 1982; Kirkpatrick-Steger, Wasserman, \& Biederman, 1996; Ushitani, Fujita, \& Yamanaka, 2001; Watanabe, 1988).

The object-line effect is a striking and robust example of a difference in categorization of forms by humans and pigeons. Pomerantz, Sager, and Stoever (1977) found that reaction times among humans discriminating between lines tilted to the left or right of vertical were twice as long when the lines were presented alone as when each line was accompanied by an L shape. An explanation proposed for this "object-line superiority effect" is that the diagonal lines embedded in identical L-shaped forms produce compound patterns that are readily nameable and therefore easier to tell apart: An L added to the lefttilted line looks like a triangle, and an $\mathrm{L}$ added to the right-tilted line looks like an arrow. Facilitation of discrimination by the context in which stimuli appear has been referred to as a "redundancy gain" by Enns and Prinzmetal (1984). They showed (Experiment 2) that the "line-context correlation was perceptually useful only when the lines-in-context were more dissimilar from each other than the target lines alone" (p. 30).

The object-line superiority effect is not found in pigeons. In an experiment in which pigeons were required to choose between two response disks according to the pattern shown on a computer monitor, Donis and Heinemann (1993) found that pigeons are consistently more accurate when the tilted lines are shown alone than when each line is embedded in an $L$ shape. For pigeons, the added context is detrimental to the performance of the task. Kelly and 
Cook (2003) obtained similar results when these forms were part of a texture display and when the pigeons' task was to decide whether sets of items were the same or different. In a human version of the latter task, these investigators found once again that the added context enhances discrimination of tilted lines by humans.

Donis and Heinemann (1993) accounted for their pigeon data using the Natural Intelligence Model (NIM) originally developed by Heinemann (1983a, 1983b) and Chase (1983) to account for identification and categorization of diffuse stimuli such as light and sound intensities. This model was later extended to account for temporal discriminations (Heinemann, 1984) as well as categorization of outline drawings (Heinemann \& Chase, 1990). According to the NIM, when identifying outline drawings, identical (and therefore noninformative) elements, such as the added L shape, are confused with the distinctive elements, an effect that decreases with increasing distance between the distinctive and common elements (Donis, Heinemann, \& Chase, 1994). The NIM is expressed in the form of a computer program that enables us to define the variables postulated to control the observed behavior.

If, as we tentatively assume, the NIM applies to pattern recognition in humans as well as pigeons, humans should also make more errors in the presence of identical contexts. For humans, the powerful influences of language may compensate for the detrimental effects of noninformative elements on discriminability when these elements create a meaningful and thus nameable object (Pomerantz, 1991). Of course, it is also possible that the differences between humans and pigeons reflect differences in early processing of visual patterns by the two species. For example, the addition of the $\mathrm{L}$ shape creates angles and line junctions that may be processed differently by humans and pigeons. Moreover, the oblique lines in the object-line patterns are mirror images of each other. Although both pigeons and humans show mirrorimage confusions (Donis, 1999; Todrin \& Blough, 1983), the addition of the L shape may be more helpful for humans than for pigeons if mirror-image confusions are more pronounced in humans, as suggested by Hollard and Delius (1982). Our understanding of these issues will be enhanced by an examination of the effects on categorization of additional noninformative context when the possible effects of mirror-image confusions and line junctions have been ruled out.

The stimuli to be categorized in the present experiments consisted of outline drawings of human faces that differed only in the expression of the mouth, which was either smiling or sad. The target stimuli, the mouths, were presented both alone and with various degrees of facial context. Whether alone or in context, the expressions of the mouth were the facial characteristics that were differentially associated with reinforcement. The required response was a choice between two illuminated response disks that differed only in position.

The outline drawings of faces were analogous to the object-line patterns described above. In both, the defining difference is a single feature-the orientation of a mouth or the tilt of a vertical line. For humans, the orientation of a mouth signifies "happiness" or "sadness." Such a "perceptual Gestalt" (Homa, Haver, \& Schwartz, 1976) may enhance performance in much the same way that naming the object-line forms enhances performance for these stimuli. McKelvie $(1973,1976)$ suggested that the role of specific facial features in categorization by humans is to make schematic faces more meaningful and thus easier to label. Even with schematic facial forms (Davidoff, 1986), a facial feature is identified more readily by humans when seen as part of a face than when seen in isolation or in an inappropriate location (Farah, Tanaka, \& Drain, 1995; Tanaka \& Farah, 1991, 1993).

For the pigeon, the added facial context is probably as meaningless as the tilted lines embedded in the L shape. Even with much more realistic stimuli than the outline drawings used here (i.e., photographs of people's faces expressing different emotions), Jitsumori and Yoshihara (1997) concluded that although pigeons showed a high level of recognition transfer to novel faces showing the same emotions, they achieved this transfer by additive integration of individual features rather than by perceiving differences in the emotion expressed. Thus, the added facial context should not enhance discriminability for pigeon observers. Rather, if the pattern recognition process described by the NIM is correct, the added context should result in a decrement in discrimination performance. Our experiments, therefore, provide an opportunity to determine whether the detrimental effects of added identical elements on pattern recognition by pigeons are or are not unique to tilted lines.

\section{Theoretical Considerations: Description of the Natural Intelligence Model}

Successful performance of a discrimination task requires attention to the relevant stimuli and correct use of this stimulus information. Unless the task is easy, acquisition of a discrimination involves a presolution period (PSP), an initial period in which there is no evidence that the discriminative stimuli control behavior, followed by a gradual increase in the proportion of correct choices. During the first (PSP) stage of learning, the organism discovers which of the numerous stimuli present when a response is reinforced is correlated with its behavior. During the second stage, this information gradually replaces the less informative information that guided behavior during the PSP. Heinemann (1983b), in an analysis of the PSP based on Wald's (1947) sequential probability ratio test, showed that the length of the PSP is a function of the discriminability of the stimuli: the more similar the stimuli to be identified, the longer the period of chance performance.

According to the NIM, the acquisition of a discrimination following the end of the PSP consists of replacing information that is irrelevant to the discrimination with relevant information. The information that guides behavior is stored and retrieved from a relatively long-term memory of limited capacity that we refer to as exemplar memory. The rate of acquisition is a function of the num- 
ber of records in this exemplar memory. For the simulations later in this article, the size of exemplar memory was fixed at 2,000 records.

The NIM provides a quantitative account of learning using conventions adopted from signal detection theory. Sensory information is represented as differences in the sensations associated with the discriminative stimuli by the distances, $d^{\prime}$, between the means of normal distributions. Unlike the criterion rule of signal detection theory, however, response selection (for the pigeons here, the response disk pecked) is based upon a comparison of the current sensation(s) to a small number of remembered events held in working memory when a decision is required. These records contain information about the sensation and the response that was reinforced. The decision made is the one that, given the information in the sample, is most likely to be correct (i.e., to result in delivery of the reinforcer). Individual pigeons appear to differ in the number of records held in working memory as well as in their sensitivity to stimulus differences.

In applying the decision scheme described above to outline drawings formed by dot-matrix patterns, such as the stimuli used in the present experiments, each dot is treated as the mean of a bivariate normal distribution, with the horizontal and vertical coordinates of the dots defining the pattern. Similarity is defined in terms of probability that each dot on the pattern represents the stimulus to be identified. The probability of a correct decision is primarily a function of the arrangement of the dots forming the patterns. The presence of identical elements increases similarity, an effect that is most pronounced when these elements are close to the distinguishing elements of the pattern (Donis et al., 1994).

In applying the model to pattern recognition, it is necessary to specify how the patterns to be compared are superimposed. According to the NIM, how patterns are viewed affects how they are remembered. In our experiments, each pigeon was required to place its head approximately where the center of the U shape, the target, would appear. Therefore, for our simulations, the pattern to be identified was compared with the remembered patterns by superimposing the patterns with their centers at this point.

The NIM is described in more detail by Chase and Heinemann (2001), in an online article containing an executable program that can be used to examine the decision process using Donis and Heinemann's (1993) stimuli. A mathematical treatment of the decision process may be found in Heinemann and Chase (1990), and for a comparison of the model with classic signal detection theory, see Chase and Heinemann (1991).

\section{EXPERIMENT 1}

In Experiment 1, we investigated the effect of adding noninformative context on discrimination of smiling or sad mouths by pigeons, using a between-subjects design. According to the NIM, the addition of facial context should be detrimental to the pigeons' discriminations of the target patterns. The detrimental effects of the nonin- formative facial contexts should not be ameliorated by the use of verbal labels or a perceptual gestalt if as we assume, this context is meaningless to pigeons. By training separate groups of pigeons on each of three pairs of stimuli that differed only in their degrees of added identical context, we were able to examine the development of discrimination among our subjects without the possible influence of exposure to the other stimuli.

\section{Method}

Subjects. The subjects were 12 experimentally naive White Carneau pigeons (Columba livia), 6 male and 6 female, purchased, as were the pigeons used in Experiment 2, from the Palmetto Pigeon Plant (Sumter, SC). All pigeons were 12 months old at the start of training and were maintained throughout the experiments at $80 \%$ of the mean free-feeding body weight that they had attained over a previous 6-week period of free access to food. The pigeons were housed individually in standard pigeon cages in a windowless colony room that was illuminated with fluorescent lamps on a 12:12-h light:dark cycle. In this colony room, the pigeons had free access to water and grit. They were given food in addition to that obtained during the experiment only if their weight fell below $80 \%$.

Apparatus. The apparatus consisted of one large environment cubicle (Model LEC-006, BRS/LVE, Beltsville, MD) divided into two chambers. The pigeons were trained and tested in the smaller of the two chambers, the walls of which were painted matte black. The interior dimensions of this chamber were $51 \mathrm{~cm}$ high $\times 57 \mathrm{~cm}$ wide $\times 46 \mathrm{~cm}$ deep. The wall dividing the two chambers had a $23 \times$ $28 \mathrm{~cm}$ opening covered by a transparent sheet of Lexan, in front of which was a position sensor system (touch screen, Model 1210, BFANM Corp., Albuquerque, NM). This device consisted of pairs of emitters and detectors that were arranged to form a grid of infrared light beams ( 20 horizontal and 32 vertical beams). The grid was scanned every $8 \mathrm{msec}$ to determine the position of any pair of crossed infrared beams interrupted by the pigeon's head. Behind the sheet of Lexan was the flat viewing screen of a Zenith color monitor (Model ZCM-1495). Another sheet of Lexan, $15 \mathrm{~cm}$ high, was placed in front of the touch screen and $3 \mathrm{~cm}$ away from the infrared emitters to prevent the pigeon from interrupting the infrared beams with its chest. Two small plastic food cups were placed below the lower edge of the frame, one to the right and one to the left. Two pellet dispensers (Model ENV-203, MED Associates, Georgia, VT) mounted on the roof of the chamber delivered food pellets (45-mg Noyes, Research Diets, Inc., New Brunswick, NJ) through a vinyl tube into the food cups. Above each of the cups there was a small amber light that could be illuminated. Experimental contingencies, generation of the stimuli, and recording of the data were controlled by a Zenith Z-425S + personal computer equipped with an interface unit (A-Bus, Alpha Products, Flanders, NJ).

Stimuli. Three pairs of stimuli were used and are shown in Figure 1. The first pair consisted of two U-shaped target patterns, $9 \mathrm{~mm}$ wide $\times 5 \mathrm{~mm}$ high, representing a smiling and a sad mouth. The second pair consisted of the same target patterns, to which three dots in an inverted triangular formation were added, representing a nose and eyes. The nose dot was located $3 \mathrm{~mm}$ above the center of the mouth and was $1 \mathrm{~mm}$ in diameter. The eye dots, each $1 \mathrm{~mm}$ wide $\times 2 \mathrm{~mm}$ high, were located $2 \mathrm{~mm}$ above the nose, with a separation of $3 \mathrm{~mm}$. The third pair of stimuli consisted of the two previous patterns embedded in an oval form. The oval was $21 \mathrm{~mm}$ wide $\times 30 \mathrm{~mm}$ high. The visual angle subtended by the width of the largest stimulus was $15.4^{\circ}$, and the height was $21.49^{\circ}$, if it is assumed that the pigeon's head was $80 \mathrm{~mm}$ from the display (the F1 position, as described by Goodale, 1983).

These stimuli were presented on the computer monitor at a $320 \times$ 200 screen resolution. The stimuli appeared white to a human observer and had a luminance of $12.9 \mathrm{~cd} / \mathrm{m}^{2}$ as measured by a Minolta 

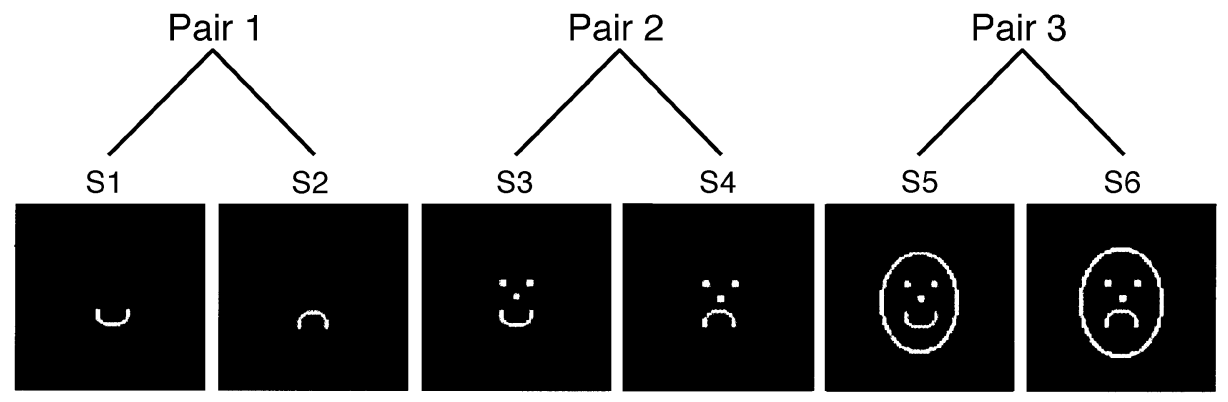

Figure 1. The three pairs of stimuli used in Experiments 1 and 2.

LS-110 luminance meter equipped with a close-up lens. The stimuli were presented in the dark chamber on the dark screen of the computer monitor (the luminance of which was below readability by the luminance meter).

Preliminary training. Following experience eating from the food cups, the pigeons were autoshaped to peck on a white disk $(1.5 \mathrm{~cm}$ in diameter) that appeared in the center of the viewing screen. When a peck was made, a beep sounded (a $50-\mathrm{msec}$ sound at $2.8 \mathrm{~Hz}$ ), followed by delivery of two food pellets at random into either the left or right food cup. The food cup into which the pellet was delivered was illuminated for $7 \mathrm{sec}$. After the pigeons were pecking reliably on the disk, a color discrimination task was introduced - that is, either a red or yellow disk was now presented. Pecks on the disk produced two light blue response disks (described below in greater detail). Pecks on the left response disk were reinforced with food if the yellow disk had been presented, and pecks on the right disk were reinforced if the red disk had been presented. Following an error, the trial was repeated. The size of the colored disk was gradually reduced over seven sessions to $1 \mathrm{~mm}$ in diameter. This $1-\mathrm{mm}$ stimulus marked the center of the $1.5 \times 1.5 \mathrm{~cm}$ area over which pecks were sensed. This region is referred to below as the active region.

Discrimination training. The 12 pigeons were randomly assigned to three groups balanced for sex. The pigeons in Group 1 were trained to discriminate between the two patterns referred to here as stimulus Pair 1; those in Group 2 were trained with Pair 2, and those in Group 3 with Pair 3 (see Figure 1).

For the first four sessions, at the start of each trial, a white dot ( $1 \mathrm{~mm}$ in diameter) appeared in the center of the active region on the darkened screen. A beep sounded and the dot disappeared when a peck was made within the active region. After $100 \mathrm{msec}$, one of the stimuli flashed on the screen for $80 \mathrm{msec}$. The stimulus was aligned so that the center point of the U-shaped form was in the center of the active region - that is, in the place where the dot had previously appeared. This was true for all six stimuli. The brief exposure to the stimulus and its presentation when the bird's head was within the active region provided control over the way the stimulus was observed (Bloch \& Martinoya, 1982; Yamashita, 1991; Zeigler, Levitt, \& Levine, 1980). At stimulus offset, two light blue response disks (1.5 $\mathrm{cm}$ in diameter) appeared to the left and right of center, with a separation of $6.5 \mathrm{~cm}$ and $0.5 \mathrm{~cm}$ above the uppermost part of the largest stimuli (Pair 3). For the three groups of pigeons, a peck on the right response disk was designated as the correct response for one stimulus of the pair, the odd-numbered stimuli in Figure 1, and a peck on the left response disk was correct for the other, the even-numbered ones in Figure 1. Correct responses were followed by the beep, illumination of the food cup on the side corresponding to the response disk that was pecked, and delivery of three food pellets. The food cup remained illuminated during the 5 -sec intertrial interval (ITI). Incorrect responses were followed by a change in the appearance of the monitor from completely dark to dark gray (a luminance of $0.39 \mathrm{~cd} / \mathrm{m}^{2}$ ) during the ITI, and afterward the same stimulus was presented again, until the correct response disk was pecked and food was delivered. Only responses made to the first presentation of a stimulus were included in the analysis of the data. Each training session consisted of 100 discrete trials, with the constraint that each stimulus in a pair was presented at random on 50 trials.

The pigeons were trained for an additional 76 sessions. These sessions were identical to the first 4, except that the 1-mm dot appeared only on the first trial of each session. On subsequent trials, following the ITI, the screen became completely dark, and the trial was initiated when the bird's head was in front of the active region as sensed by the photocell beams. It was observed that the pigeons used the illumination from the amber light above the food cup during the ITI to position their heads at the center of the screen - the active region - before the start of the trial. The removal of the center dot was done to prevent the pigeons from focusing their attention exclusively on this area, thus increasing the probability that they would observe the entire stimulus. The data obtained over all 80 training sessions were used in all analyses.

\section{Results and Discussion}

The left panels of Figure 2 show the mean proportions correct for each bird as a function of training under the three conditions in Experiment 1. As expected, these data show that accuracy was best for the targets alone (Pair 1), it was reduced when dots representing the eyes and mouth were added (Pair 2), and when the oval was added (Pair 3), accuracy remained at chance throughout all 80 sessions. The mean proportions correct (p) for 5-session blocks of training (transformed to the arcsine of $\mathrm{p}^{1 / 2}$, a variance stabilizing transformation proposed by Freeman \& Tukey, 1950, for binomial data), were analyzed using a 3 (stimulus pair) $\times 16$ (block) analysis of variance (ANOVA) of mixed design. This analysis revealed a significant main effect of block $[F(15,135)=4.96, p<.001]$. The effect of stimulus pair was not significant $[F(2,9)=3.75, p>$ $.05]$. The curves showing proportions correct for the different pairs in Figure 2 diverge as a function of blocks of practice, indicative of a significant interaction between stimulus pair and block of practice $[F(30,135)=1.93$, $p<.05]$. To eliminate differences in rates of learning from the comparison of conditions, a one-way ANOVA was performed on the arcsine-transformed proportions correct for the final 20 sessions, during which accuracy appeared to have approached asymptote. The effect of stimulus pair averaged across the final 20 sessions was significant $[F(2,9)=4.86, p<.05]$. For Pair 1 , the mean proportion correct was $.714(S D=.106)$; for Pair 2 , the 

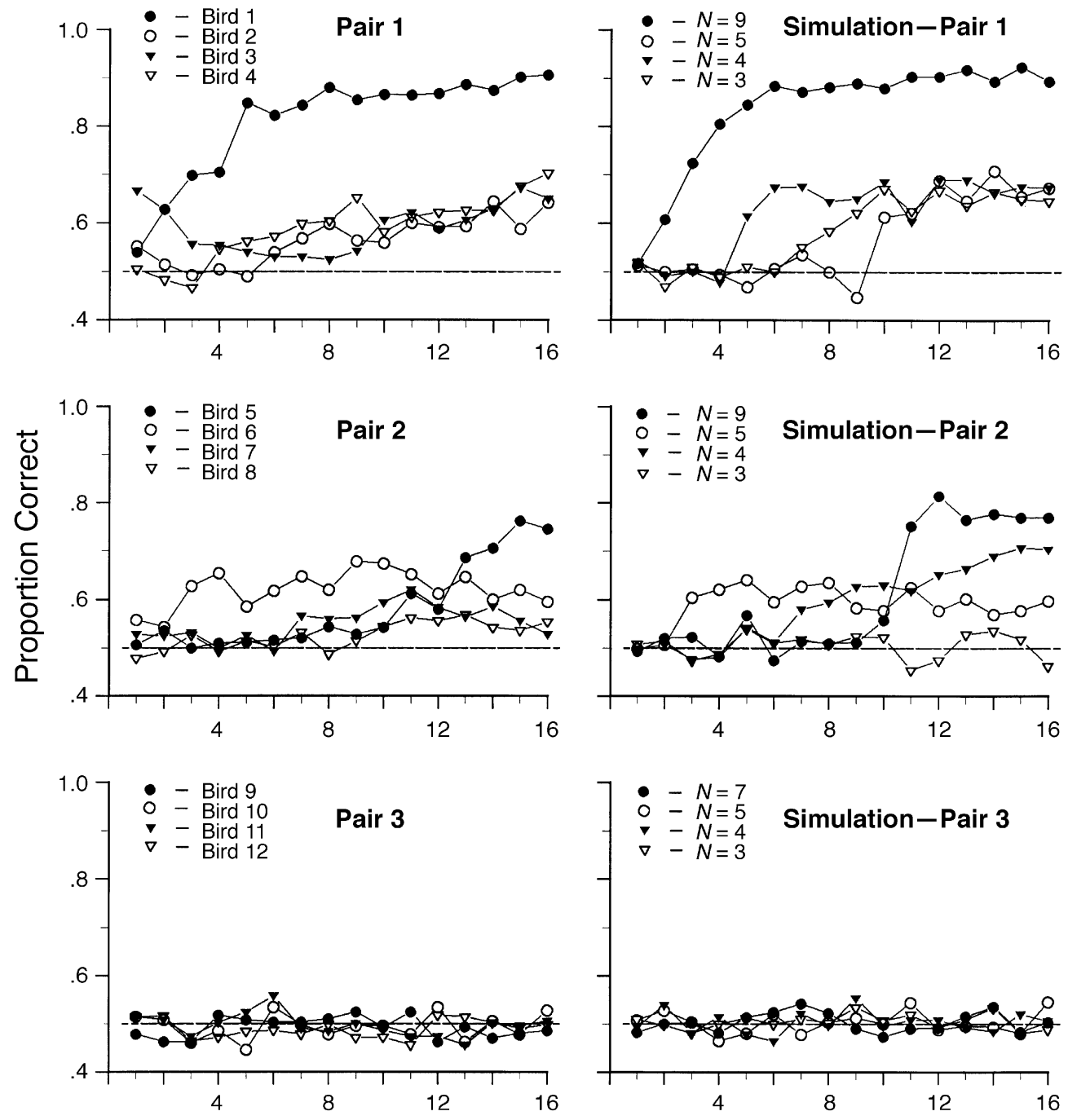

Blocks of 5 Sessions

Figure 2. The proportions correct for the pigeons trained separately on the three pairs of stimuli, as a function of blocks of five 100-trial sessions. The horizontal line at .5 represents chance performance. The left panels show the data for the 4 pigeons in each group. The right panels show simulations of performance under the various experimental conditions using the NIM for different numbers $(N)$ of records used in the decision process.

mean was .612 $(S D=.07)$; and for Pair 3, the mean was $.491(S D=.007)$. Although these differences were as expected, Tukey's HSD indicated that only the difference between Pairs 1 and 3 was significant $(p<.03)$.

The right panels in Figure 2 show the results of simulations using the NIM. These simulations are not attempts to obtain best fits to the data. Rather, they are meant to illustrate how the model performs in the presence of the three types of patterns under the present experimental conditions. Because random processes are involved in the decision, the curves are not smooth. Identical parameters yield slightly different curves each time the simulation is repeated.

For each bird, the PSP was estimated to end when the proportion correct for blocks of five sessions exceeded and remained above .55 , a level of accuracy that would rarely be exceeded by chance in the absence of discrimination. None of the birds trained with Pair 3 exceeded this level. Following the PSP, a gradual increase in accuracy was generated in the simulations by random replacement on each trial of a record that did not contain stimulus information by one that did. The differences in accuracy for Pairs 1 and 2 shown by the birds and in the simulations reflect differences in the dot-matrix patterns to be identified, and to a lesser extent the number $(N)$ of records on which each decision was based. The effects of number of records, ranging from 3 to 9 , on accuracy are shown for Pairs 1 and 2. The sensitivity parameter, the spacing of the dots forming the pattern, was fixed for all simulations except for Bird 1, whose data are approximated by the sim- 
ulation for $N=9$. This bird appeared to be more sensitive to the differences in the stimuli than were the other birds. To account for this behavioral pattern, the spacing of the dots was increased slightly in the simulations.

Of particular interest is the finding that accuracy of the pigeons trained with Pair 3 remained at chance throughout training, whereas all of the pigeons trained with Stimulus Pairs 1 and 2 eventually performed above chance. These results provide further evidence that added noninformative context is detrimental to discrimination, as found by Donis et al. (1994) and in keeping with Heinemann's (1983b) suggestion that the more difficult the discrimination task, the longer the period of chance performance.

\section{EXPERIMENT 2}

The effect of identical context on the discrimination of inverted and upright $U$ forms was investigated further in Experiment 2, using a within-subjects design. This experiment made it possible to compare performance within individual pigeons for the three pairs of patterns. Just as in Experiment 1, the addition of identical context was expected to have detrimental effects on discrimination of the target patterns. This experiment also represented a first attempt to determine how the length of the PSP is affected by simultaneous training of three discriminations that differ in difficulty.

\section{Method}

Subjects. The subjects were 5 experimentally naive White Carneau pigeons (Columba livia), 2 male and 3 female. All pigeons were 12 months old at the start of training and were maintained throughout the experiment at $80 \%$ of their free-feeding body weight. The pigeons were kept in the same room and under the same conditions as those in Experiment 1.

Training. Preliminary and discrimination training were the same as in Experiment 1, except that in this experiment all of the pigeons were trained with all six stimuli. As before, there were 80 sessions of training. Each training session consisted of 150 discrete trials, with the constraint that each of the six stimuli was presented at random on 25 trials. For all pigeons, a peck on the right response disk was correct for the odd-numbered stimuli in Figure 1, and a peck on the left response disk was correct for the even-numbered stimuli.

\section{Results and Discussion}

Two of the pigeons' accuracy remained at chance for all six stimuli. It appears that with the presence of Pair 3 on one third of the trials, these pigeons did not emerge from the PSP even for the "easier" discrimination tasks (Pairs 1 and 2). The data for these 2 pigeons are not included in the following statistical analyses. The left panels in Figure 3 show the mean proportions correct as a function of training for the 3 pigeons in Experiment 2 that performed above chance with stimulus Pairs 1 and 2. Once again, it is clear that the addition of identical noninformative context was detrimental to discrimination of the target patterns. The mean proportions correct for 5-session blocks of training (transformed to the arcsine of $\mathrm{p}^{1 / 2}$ ) were analyzed using a 3 (stimulus pair) $\times 16$ (block) ANOVA with repeated measures. This analysis revealed a significant main effect of block $[F(15,30)=3.63, p<.001]$, but the effect of stimulus pair was not significant $[F(2,4)=3.85$, $p>.05]$. As in Experiment 1, the curves showing proportions correct for the different pairs in Figure 3 diverge as a function of practice. There was a significant interaction between stimulus pair and practice $[F(30,60)=2.88, p<$ $.001]$. To eliminate differences in rates of learning from the comparison of conditions, a one-way ANOVA was performed on the arcsine-transformed proportions correct for the final 20 sessions, during which accuracy was relatively stable. When averaged across birds, the proportions correct for these 20 sessions were $.75(S D=.074)$ for Pair 1, .661 $(S D=.047)$ for Pair 2, and $.499(S D=.007)$ for Pair 3. The effect of stimulus pair was significant $[F(2,4)=23.94, p<.01]$. Paired sample $t$ tests showed significant differences between Pairs 1 and $3(p=.04)$ and Pairs 2 and $3(p=.032)$.

The right panels of Figure 3 show the results of performance simulations of this task using the NIM. The PSP was estimated from the data using the same procedure as in Experiment 1. The data and the simulations show once again that added identical context is associated with decreased accuracy. The acquisition of the real (left) and simulated (right) pigeons suggests that discrimination decisions were based on three records for Bird 14, six for Bird 15, and nine for Bird 17. Because the same birds were tested with all three pairs of patterns, differences in accuracy among these patterns cannot be attributed to differences in the number of records used in the decision process or in the ways different pigeons viewed the stimuli.

These data raise interesting questions about the PSP. According to Heinemann's (1983b) treatment of the PSP, the rate at which information accumulates during this period is a function of the discriminability of the stimulithe smaller the difference among the sensations associated with the discriminative stimuli, the greater the number of trials before the end of the PSP. If information regarding the sensations associated with each pair of patterns is acquired separately, the length of the PSP should vary with the discriminability of each of the stimuli, as was seen in Experiment 1. However, it is also possible that, with simultaneous training on discriminations differing in difficulty, as was done here, the PSP depends upon the average difficulty. If so, the length of the PSP would be equivalent for the three pairs of stimuli. The failure of 2 pigeons in this experiment to show evidence of learning suggests that trials with the more difficult discrimination (Pair 3) may lengthen the PSP for the more discriminable patterns (Pairs 1 and 2) as well. Would these birds have learned if training had continued? The bird that showed the best discrimination, Bird 17, may have begun to emerge from the PSP for Pair 3 during the final blocks of training. Clearly, further work is needed with simultaneous training on patterns differing in difficulty in order to determine whether the length of the PSP is a function of the overall difficulty of a discrimination or whether it is determined by the discriminability of each of the patterns. 

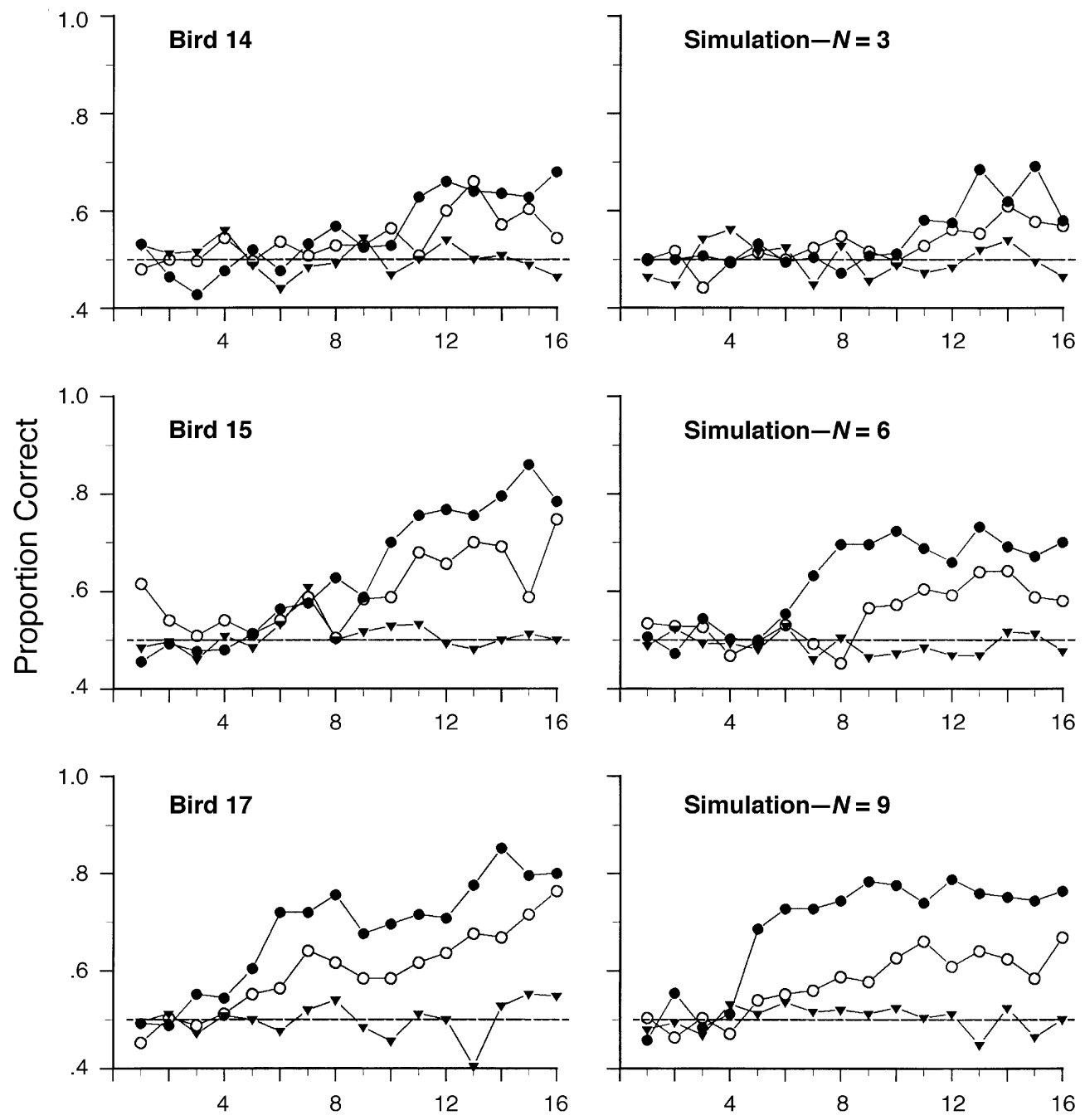

$$
\begin{aligned}
& \text { - Pair } 1 \\
& \text { - - Pair } 2 \\
& \text { - - Pair } 3 \\
& \text { - - - Chance }
\end{aligned}
$$

Figure 3. The proportions correct for the pigeons trained on the three pairs of stimuli simultaneously, as a function of blocks of five 150-trial sessions. The horizontal line at .5 represents chance performance. The left panels show proportions for the 3 pigeons in this experiment that exceeded chance performance. The right panels show simulations of performance according to the NIM.

\section{GENERAL DISCUSSION}

These findings show once again that pigeons are best at identifying patterns that do not have identical elements, such as the added facial context used here or the $\mathrm{L}$ shape in object-line experiments. As predicted, the addition of identical elements to target patterns decreases discrimination accuracy. The results of this study, therefore, show that such a reduction in accuracy is not limited to experiments using straight lines, angles, or mirror-image confusions. There is a systematic increase in the length of the PSP, as well as in the number of errors, as a function of the degree to which patterns include identical elements. This effect was shown both for discriminations trained separately (Experiment 1) and for those trained simultaneously (Experiment 2).

The results of the present experiments are in sharp contrast to the object-line and facial superiority effects found with human participants (e.g., Pomerantz, 1991; Tanaka $\&$ Farah, 1993). The detrimental effects of identical noninformative context on discrimination accuracy among pigeons have now been demonstrated in two situations in which humans benefit from the added context. For humans, 
these stimuli are readily coded into words - the objectline patterns are a triangle or an arrow, the faces are sad or happy. To account for the ambiguous effects of verbal codes on increasing or decreasing discriminability of forms (the acquired distinctiveness or equivalence of cues), Epstein (1967) pointed out that participants often ignore experimenter-supplied labels or create more effective labels of their own. Data such as these suggest that processes such as visual pattern recognition may best be studied in organisms that cannot use linguistic coding to increase the discriminability of stimuli.

As a theoretical framework for the treatment of learning, the NIM enables us to provide a quantitative description of discrimination acquisition involving choice between two or more alternatives. The simulations shown in Figures 2 and 3 make possible a comparison of data generated by the pigeons and by the model. The length of the PSP was estimated from the real-pigeon data. In order to obtain the proportions correct in the absence of stimulus information, the simulations were done with records containing only response information. An equal number of records in memory represented each response, as would be the state of exemplar memory if, during shaping, the birds' choices of the two disks were reinforced equally often. Following the PSP, each correct response was accompanied by replacement of a record in memory by a record containing information about the stimulus found to be relevant during the PSP and the response that was reinforced. Because the record that was replaced was randomly selected, the rate at which noninformative records were replaced decreased as fewer such records remained to be replaced. This process yielded the negatively accelerated learning curve generated by virtually all theories of learning and typically observed as a function of reinforced trials. According to the NIM, the rate of acquisition is also influenced by the number of records in exemplar memory. For these simulations, we assumed an exemplar memory size of 2,000 records; had we used a smaller number, the increases in proportions correct would have been faster than those observed here.

The only other parameter manipulated in generating the simulated acquisition curves was sample size $(N)$, the number of records sampled from exemplar memory that are assumed to be used in the decision process. According to the NIM, individuals differ in the amount of information that they can process simultaneously - that is, in the capacity of working memory. Simulations suggest that the pigeons that made the fewest errors (Birds 1 and 5 in Experiment 1 and Bird 17 in Experiment 2) based their choices on nine records, whereas the birds that made the most errors (Birds 2 and 8 in Experiment 1 and Bird 14 in Experiment 2) based their choices on three records. Thus, according to the NIM, the number of records on which a decision is based is primarily responsible for differences among individuals trained with the same stimuli. Interestingly, differences in sample size may also be responsible for differences among species in psychophysical tasks, such as absolute identification of light intensities (Chase \& Heinemann, 1991).
The simulations yielded the same ordering in proportions correct as that found for the pigeons, as a function of the degree of noninformative context in the patterns. This equivalence is seen most clearly in the simulated curves for Experiment 2, shown to the right in Figure 3. In this experiment, the real and simulated discriminations of the target stimuli, the inverted and upright $U$ shapes embedded in their two degrees of noninformative context, may be compared directly. By Block 16, for Pair 3, the proportions correct were still close to chance; for Pair 2, accuracy was above chance; and for Pair 1 (the targets alone), accuracy was highest. These effects were observed for sample sizes of three, six, and nine records. Thus, the differences in accuracy shown by both the real and the simulated birds are due to the patterns to be discriminated.

The prediction that common elements will decrease discriminability is not new. In fact, it is generally expected that similarity will be increased by the addition of such elements (e.g., in the work with humans on acquired equivalence reviewed by Epstein, 1967, and in the work with pigeons by Pearce \& Redhead, 1993). According to most exemplar models of pattern recognition in human learning (e.g., those of Estes, 1994; Kruschke, 1992; Medin \& Schaffer, 1978; and Nosofsky, 1986) and in animal learning (e.g., that of Pearce, 1994), similarity is defined in terms of the number of common features. The NIM provides an alternative to such feature models by describing an algorithm for computation of similarity that does not require the extraction and identification of features. Instead, the NIM assumes that the patterns remain intact. This model has implications for the effects of manipulations such as those described by Kirkpatrick-Steger, Wasserman, and Biederman (1996, 1998), in which parts of outline drawings are deleted or moved. According to the NIM, changes in shapes as well as in the locations of the shapes should affect accuracy, as indeed they do (Kirkpatrick-Steger, Wasserman, \& Biederman, 2000; Van Hamme, Wasserman, \& Biederman, 1992).

The research presented here is a step toward understanding visual pattern recognition by pigeons. As such, it is directly relevant to the question raised in the introduction to this article: How do pigeons categorize complex visual patterns, which may be photographic exemplars of classes humans identify by name, such as trees, fish, or water? Although much remains to be done before such complex stimuli can be subjected to the type of analyses we were able to do here with dot-matrix patterns, models such as the NIM that treat categorization of patterns as a process of comparing the pattern to be identified with individual exemplars held in memory may ultimately provide an answer to the question that has intrigued psychologists following Herrnstein and Loveland's (1964) discovery of the remarkable ability of pigeons to categorize pictures. If the NIM is correct, this categorization is accomplished without the creation of prototypes or the extraction of features. We have shown that the NIM accounts for the effects of noninformative common elements on discrimination. However, other models do so as well. Additional work is needed, with different patterns and under 
different conditions, before sufficient evidence accumulates for understanding of these processes to emerge from its own presolution period.

\section{REFERENCES}

Bloch, S., \& Martinoya, C. (1982). Comparing frontal and lateral viewing in the pigeon: I. Tachistoscopic visual acuity as a function of distance. Behavioural Brain Research, 5, 231-244.

Blough, D. S., \& Blough, P. M. (1997). Form perception and attention in pigeons. Animal Learning \& Behavior, 25, 1-20.

Chase, S. (1983). Pigeons and the magical number seven. In M. L. Commons, R. J. Herrnstein, \& A. R. Wagner (Eds.), Quantitative analyses of behavior: Vol. 4. Discrimination processes (pp. 37-57). Cambridge, MA: Ballinger.

Chase, S., \& Heinemann, E. G. (1991). Memory limitations in human and animal signal detection. In M. L. Commons, J. A. Nevin, \& M. C. Davison (Eds.), Signal detection: Mechanisms, models, and applications. Hillsdale, NJ: Erlbaum.

Chase, S., \& Heinemann, E. G. (2001). Exemplar memory and discrimination. In R. G. Cook (Ed.), Avian visual cognition. Retrieved April 20, 2005 from www.pigeon.psy.tufts.edu/avc/chase/.

Соoк, R. G., Саvoto, K. K., \& Cavoto, B. R. (1996). Mechanism of multidimensional grouping, fusion, and search in avian texture discrimination. Animal Learning \& Behavior, 24, 150-167.

DAvidoff, J. (1986). The mental representation of faces: Spatial and temporal factors. Perception \& Psychophysics, 40, 391-400.

Donis, F. J. (1999). The oblique effect in pigeons ( $\overline{C o l u m b a ~ l i v i a) . ~ J o u r-~}$ nal of Comparative Psychology, 113, 107-115.

Donis, F. J., \& Heinemann, E. G. (1993). The object-line inferiority effect in pigeons. Perception \& Psychophysics, 53, 117-122.

Donis, F. J., Heinemann, E. G., \& Chase, S. (1994). Context effects in visual pattern recognition by pigeons. Perception \& Psychophysics, 55, 676-688.

ENNS, J. T., \& Prinzmetal, W. (1984). The role of redundancy in the object-line effect. Perception \& Psychophysics, 35, 22-32.

EpsteIn, W. (1967). Varieties of perceptual learning. New York: McGrawHill.

Estes, W. (1994). Classification and cognition. Oxford: Oxford University Press.

Farah, M. J., Tanaka, J. W., \& Drain, H. M. (1995). What causes the face inversion effect? Journal of Experimental Psychology: Human Perception \& Performance, 21, 628-634.

FreEman, M. F., \& TukeY, J. W. (1950). Transformations related to the angular and the square root. Annals of Mathematical Statistics, 21, 607-611.

GoODALE, M. A. (1983). Visually guided pecking in the pigeon (Columba livia). Brain, Behavior, \& Evolution, 22, 22-41.

Heinemann, E. G. (1983a). A memory model for decision processes in pigeons. In M. L. Commons, R. J. Herrnstein, \& A. R. Wagner (Eds.), Quantitative analyses of behavior: Vol. 4. Discrimination processes (pp. 3-19). Cambridge, MA: Ballinger.

HeinemanN, E. G. (1983b). The presolution period and the detection of statistical associations. In M. L. Commons, R. J. Herrnstein, \& A. R. Wagner (Eds.), Quantitative analyses of behavior: Vol. 4. Discrimination processes (pp. 21-35). Cambridge, MA: Ballinger.

HeinemanN, E. G. (1984). A model for temporal discrimination and generalization. In J. Gibbon \& L. Allan (Eds.), Timing and time perception (Annals of the New York Academy of Sciences, Vol. 423, pp. 361-371). New York: New York Academy of Sciences.

HeinemanN, E. G., \& CHASE, S. (1990). A quantitative model for pattern recognition. In M. L. Commons, R. J. Herrnstein, S. M. Kosslyn, \& D. B. Mumford (Eds.), Quantitative analyses of behavior: Vol. 9. Computational and clinical approaches to pattern recognition and concept formation (pp. 109-126). Hillsdale, NJ: Erlbaum.

Herrnstein, R. J., \& Loveland, D. H. (1964). Complex visual concept in the pigeon. Science, 146, 549-551.

Hollard, V. D., \& DeliUs, J. D. (1982). Rotational invariance in visual pattern recognition by pigeons and humans. $\underline{\text { Science, }}, \underline{\mathbf{2 1 8}}, \underline{804-}$ 806.

Homa, D., Haver, B., \& Schwartz, T. (1976). Perceptibility of schematic face stimuli: Evidence for a perceptual gestalt. Memory \& Cognition, 4, 176-185.

HUBBer, L. (2001). Visual categorization in pigeons. In R. G. Cook (Ed.), Avian visual cognition. Retrieved April 20, 2005 from www.pigeon. psy.tufts.edu/avc/huber/.

JITSUMORI, M., \& YOSHIHARA, M. (1997). Categorical discrimination of human facial expressions by pigeons: A test of the linear feature model. Quarterly Journal of Experimental Psychology, 50B, 253-268.

Kelly, D. M., \& CoOK, R. G. (2003). Differential effects of visual context on pattern discrimination by pigeons (Columba livia) and humans (Homo sapiens). Journal of Comparative Psychology, 117, 200-208.

KirkPATrick-Steger, K., Wasserman, E. A., \& Biederman, I. (1996). Effects of spatial rearrangement of object components on picture recognition in pigeons. Journal of the Experimental Analysis of Behavior, 65, 465-475.

KiRKPATRICK-STEGER, K., WASSERMAN, E. A., \& Biederman, I. (1998). Effects of geon deletion, scrambling, and movement on picture recognition in pigeons. Journal of Experimental Psychology: Animal Behavior Processes, 24, 34-46.

KirkPATRICK-STEgeR, K., Wasserman, E. A., \& Biederman, I. (2000). The pigeon's discrimination of shape and location information. $\mathrm{Vi}$ sual Cognition, 7, 417-436.

KRUSCHKE, J. K. (1992). ALCOVE: An exemplar-based connectionist model of category learning. Psychological Review, 99, 22-44.

McKelvie, S. J. (1973). The meaningfulness and meaning of schematic faces. Perception \& Psychophysics, 14, 343-348.

McKelvie, S. J. (1976). The effects of verbal labelling on recognition memory for schematic faces. Quarterly Journal of Experimental Psychology, 28, 459-474.

Medin, D. L., \& Schaffer, M. M. (1978). Context theory of classification learning. Psychological Review, 85, 207-238.

Nosofsky, R. M. (1986). Attention, similarity, and the identificationcategorization of integral stimuli. Journal of Experimental Psychology: Learning, Memorv, \& Cognition, 13, 87-108.

PEARCE, J. M. (1994). Similarity and discrimination: A selective review and connectionist model. Psychological Review, 101, 587-607.

Pearce, J. M., \& Redhead, E. S. (1993). The influence of an irrelevant stimulus on two discriminations. Journal of Experimental Psychology: Animal Behavior Processes, 19, 180-190.

PomerantZ, J. R. (1991). The structure of visual configurations: Stimulus versus subject contributions. In G. R. Lockhead \& J. R. Pomerantz (Eds.), The perception of structure (pp. 195-210). Washington, DC: American Psychological Association.

Pomerantz, J. R., Sager, L. C., \& Stoever, R. J. (1977). Perception of wholes and of their component parts: Some configural superiority effects. Journal of Experimental Psychology: Human Perception \& Performance, 3, 422-435.

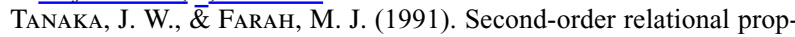 erties and the inversion effect: Testing a theory of face perception. Perception \& Psychophysics, 50, 367-372.

TANAKA, J. W., \& FARAH, M. J. (1993). Parts and wholes in face recognition. Quarterly Journal of Experimental Psychology, 46A, 225-245.

Todrin, D. C., \& Blough, D. S. (1983). The discrimination of mirrorimage forms by pigeons. Perception \& Psychophysics, 34, 397-402.

Ushitani, T., Fujita, K., \& Yamanaka, R. (2001). Do pigeons (Columba livia) perceive object unity? Animal Cognition, 4, 153-161.

Van Hamme, L. J., Wasserman, E. A., \& Biederman, I. (1992). Discrimination of contour-deleted images by pigeons. Journal of Experimental Psychology: Animal Behavior Processes, 18, 387-399.

WALD, A. (1947). Sequential analysis. New York: Dover.

Watanabe, S. (1988). Failure of visual prototype learning in the pigeon. Animal Learning \& Behavior, 16, 147-152.

Yamashita, H. (1991). Frontal visual field position and visual discrimination learning in the pigeon (Columba livia). (Doctoral dissertation, City University of New York). Dissertation Abstracts International, 52, 1104.

Zeigler, H. P., LeVITT, P. W., \& Levine, R. R. (1980). Eating in the pigeon (Columba livia): Movement patterns, stereotypy, and stimulus control. Journal of Comparative \& Physiological Psychology, 94, 783-794.

(Manuscript received September 25, 2003; revision accepted for publication August 16, 2004.) 